\title{
Incidence and Early Outcome of Coagulopathy among Major Trauma Patients
}

Daniel Ojuka, Josiah Mwendwa, Peter Odhiambo

Department of Surgery, University of Nairobi

Correspondence to: Dr. Daniel Ojuka, P.O. Box 19762-00202, Nairobi, Kenya. Email:danielojuka@gmail.com

\section{Abstract}

Background: Despite improvement in many aspects of acute trauma care, uncontrolled bleeding is responsible for more than $50 \%$ of all trauma related deaths within the first two days of admission. Objective: This study sought to determine the incidence of coagulopathy among major trauma patients and their associated outcome. Methods: A prospective descriptive study carried at $\mathrm{KNH}$ among major trauma patients. Coagulation status was determined within 30 minutes of arrival. Outcomes assessed included length of hospital stay, transfusion requirement and mortality for 30 days. Fischer's exact test was used to analyze categorical variables and Student $T$ test used to analyze continuous variables. Results: Majority (85.7\%) of the 140 patients recruited were male and, the mean age was 29.4 years $(\mathrm{SD}=12.0)$. Coagulopathy was present in $52.1 \%$ of the patients. The mean ISS on admission was 23.3 $(\mathrm{SD}=4.8)$, patients with coagulopathy had a higher ISS $22.2(\mathrm{SD}=5.2, \quad \mathrm{p}=0.018)$. The patients with coagulopathy stayed longer in hospital 12 days and their mortality was higher $21(95.5 \%) \mathrm{p}<0.001$. Conclusion: Majority of trauma patients were coagulopathic. Initial assessment of coagulation status in trauma patients is useful in planning care and anticipating complications.

Key words: Coagulopathy, Major trauma, Incidence, Outcomes, Low and middle income countries

Ann Afr Surg. 2018; 15(1):20-24

DOI:http://dx.doi.org/10.4314/aas.v15i1.5

(C) 2018 Author. This work is licensed under the Creative Commons Attribution 4.0 International License

\section{Introduction}

Uncontrolled bleeding accounts for more than half of all trauma related deaths within the first 2 days of admission (1). The six main mechanisms of coagulopathy in trauma include shock, tissue damage, haemodilution, hypothermia, acidosis and inflammation $(2,3)$. Recently, Brohi et al. described the role of hypoperfusion in initiation of coagulopathy of trauma in a cohort study (4).Most studies have used variants of deranged prothrombin time (PT) and partial thromboplastin time (PTT) to determine presence of coagulopathy in trauma $(2,3,5-7)$. One study done using thromboelastometry found correlation between its components and PT and PTT as a measure of coagulopathy with additional cut-off point that determine need for transfusion (7). Patients who arrive at the emergency department with coagulopathy following trauma are up to four times more likely to die $(2,3)$, and eight times more likely to die within the first two days $(2,3,5,6)$. Coagulopathy of trauma is associated with longer intensive care unit (ICU) and hospital, increased transfusion requirement and higher chances of organ failure $(2,5,8)$. Identifying patients who are at risk of developing acute coagulopathy of trauma and their aggressive management has been shown to reduce trauma associated morbidity and mortality (5). No study has documented local prevalence of coagulopathy in patient with major trauma. Most studies are from other context from which management protocols have been drawn and these have changed the prognosis of this set of trauma patients (9). This study sought to document our local incidence of coagulopathy of trauma.

\section{Methods}

Between February and May 2015, we carried out a descriptive study among prospectively recruited major trauma patients (Injury Severity Score (ISS) $>15$ ) 
admitted into Kenyatta National Hospital (KNH) through the Accident and Emergency (A\&E) department. The major trauma patients due for admission were identified during the primary survey. The severity of injury was assessed using Abbreviated injury score (AIS) 90 and ISS methods and time sequence from injury to review in the hospital documented. For the recruited patients, blood was drawn within 30 minutes upon arrival to the A\&E then placed in a citrated or EDTA or plain laboratory specimen bottle, placed in a cool box and delivered to the laboratory within 30 minutes for analysis of PT/PTT, full blood count and renal function tests (RFTs). The handling of the blood test and blood products was performed in the normal manner by the routine staff. Demographic data and vital signs were recorded on the initial assessment at the A\&E department. We measured both PT and PTT in all the patients but used PT as the determinant of coagulopathy.

The patients were then followed up on the second, $7^{\text {th }}$, 14th and $30^{\text {th }}$ days post operatively. The following clinical outcome variables determined and recorded: blood transfusion requirements, length of critical care unit stay, presence of acute kidney injury (as defined by the treating physicians), length of hospital stay and mortality. Patients discharged from the hospital before day 30 were deemed to be survivors and further follow up was not done.

We excluded patients who had been managed at another facility prior to review in $\mathrm{KNH}$ A\&E department, patients on anticoagulants, patients with known comorbidities (hypertension, diabetes, coronary artery disease, convulsive disorder, chronic renal disease or burns) and patients who did not consent.

Data was entered into Statistical Package for the Social Sciences (SPSS) version 21 for analysis. Variables were summarized into means, medians, percentages and proportions. Multiple logistic regression analysis was used to ascertain the association between the initial coagulation parameters and the mortality.

The Kaplan Meier method was utilized for survival analysis, log rank test was used to determine the difference between the two survival curves for patients with normal versus abnormal PT/PTT to ascertain if it is statistically significant. A 'p' value $<0.05$ was considered significant. This study was approved by the institutional review board.

\section{Results}

A total of 140 major trauma patients were recruited of whom $120(85.7 \%)$ were male, giving a male: female ratio of $6: 1$. The age ranged from 3 to 80 (mean $=29.4$, $\mathrm{SD}=12.0$ ) years. There was no statistically significant difference in the mean age among the patients with coagulopathy compared to those without (Table 1).

Table 1: Socio-demographic characteristics

\begin{tabular}{|l|l|}
\hline Variable & Frequency (\%) \\
\hline Age in years & $29.4(12.0)$ \\
Mean (SD) & $3-80$ \\
Can-Max & \\
$\leq 12$ & $10(7.1)$ \\
$13-19$ & $12(8.6)$ \\
$20-29$ & $57(40.7)$ \\
$30-39$ & $38(27.1)$ \\
$40-49$ & $14(10.0)$ \\
$\geq 50$ & $9(6.4)$ \\
\hline Gender & \\
Female & $20(14.3)$ \\
Male & $120(85.7)$ \\
\hline Education & \\
No formal education & $16(11.4)$ \\
Primary education & $44(31.4)$ \\
Secondary education & $61(43.6)$ \\
Tertiary & $19(13.6)$ \\
\hline Occupation & \\
Employed & $37(26.4)$ \\
Student & $18(12.9)$ \\
Peasant & $13(9.3)$ \\
Business & $33(23.6)$ \\
None & $39(27.9)$ \\
\hline & \\
\hline
\end{tabular}

Table 2: Vital signs

\begin{tabular}{|l|l|}
\hline Variable & Frequency (\%) \\
\hline $\begin{array}{l}\text { Blood pressure (mmHg) } \\
\text { Systolic, mean (SD) }\end{array}$ & $113.7(23.4)$ \\
Categories, n (\%) & \\
$>100$ & $94(67.1)$ \\
$\leq 100$ & $46(32.9)$ \\
Diastolic, mean (SD) & $68.8(16.3)$ \\
\hline Pulse rate, mean (SD) & $102.3(26.7)$ \\
Tachycardia, n (\%) & $67(47.9)$ \\
\hline $\begin{array}{l}\text { Respiratory rate } \\
\text { (breaths/minute) means }\end{array}$ & $24.4(13.1)$ \\
(SD) & $73(52.1)$ \\
$>20, n(\%)$ & \\
\hline Temperature & $36(41.0)$ \\
Categories, n (\%) & \\
Below 35 C & $17(12.1)$ \\
Above $37.5^{0} \mathrm{C}$ & $6(4.3)$ \\
\hline
\end{tabular}


The admission vital signs are as shown in Table 2. The Glasgow Coma Scale was 15 in 68 (48.6\%), 9-14 in 58 (48.6\%) and below 8 in $14(10 \%)$ patients.

Table 3 shows the coagulation status. PT and PTT measurements were positively correlated $(\mathrm{r}=0.647(\mathrm{p}<$ 0.001). Coagulopathic patients were older (mean 30.4, $\mathrm{SD}=12.2$ years) than those without coagulopathy (mean $28.4, \mathrm{SD}=11.7$ years) but this was not statistically

Table 3: Coagulation status

\begin{tabular}{|l|l|}
\hline Variable & Frequency (\%) \\
\hline PT(seconds) & \\
Mean (SD) & $15.5(3.5)$ \\
Category, n (\%) & \\
Raised & $73(52.1)$ \\
Normal & $67(47.9)$ \\
\hline PTT (seconds) & \\
Mean (SD) & $31.7(10.9)$ \\
Category, n (\%) & \\
Abnormal & $32(22.9)$ \\
Normal & $108(77.1)$ \\
\hline
\end{tabular}

Significant $(p=0.313)$. Nearly two thirds $(62.2 \%)$ of coagulopathic patients had received fluids prior to admission. The leading causes of injury were Road Traffic injuries (RTIs) $(55 \%, \mathrm{n}=77)$ followed by assault $(17.1 \%, \mathrm{n}=24)$. Others were falls from heights $(12.1 \%$, $\mathrm{n}=17)$, Stab or cut wounds $(5.7 \%, \mathrm{n}=8)$, gun shots $(5 \%$, $\mathrm{n}=7)$ and blunt trauma $(5 \%, \mathrm{n}=7)$.

Most of the patients had injuries to more than one body region as per the AIS scoring system. Majority of patients $(65 \%, \mathrm{n}=91)$ had head injury. Injuries to external surfaces $(60 \%, \mathrm{n}=84)$, extremities $(45 \%, \mathrm{n}=63)$, thorax $(25 \%, \mathrm{n}=35)$ and abdomen $(22.1 \%, \mathrm{n}=31)$ were the next commonest. The face was the least injured $(17.9 \%, \mathrm{n}=25)$. The mean ISS on admission was 23.3 (Range 15-38, $\mathrm{SD}=4.8$ ). Patients with coagulopathy had higher mean ISS than those without $(22.2, \mathrm{SD}=5.2$ vs. 20.3, $\mathrm{SD}=4.2 . \mathrm{p}=0.0018$ ).

The average pre-hospital time was 4 (0.5-72) hours with an 'IQR of $3-8.8$ '. Only a few of the patients had received any form of intervention (intravenous fluids) prior to hospital arrival.

There were 13(9.4\%) patients who developed (Acute Kidney Injury) AKI, 5 (38.5\%) of whom had coagulopathy. The odds ratio of developing AKI in the patients with coagulopathy was $0.5(\mathrm{p}=0.286)$.
Thirty two (22.9\%) patients received whole blood transfusion of which 13,7 and 10 patients received 1, 2 and 3 units respectively. Twenty two $(68.8 \%)$ of these patients had coagulopathy. No patient received component transfusion. There was a strong correlation between patients with coagulopathy and transfusion $(\mathrm{OR}=2.5, \mathrm{p}=0.032)$.

There were 22 mortalities (15.7\%), 115 discharges $(82.1 \%)$ and 3 patients $(2.1 \%)$ still in the ward by the end of the study period. Out of the mortalities, 21 (95.5\%) were coagulopathic. This was statistically significant $(\mathrm{OR}=26.7 \quad(3.5-204.7), \mathrm{p}<0.001)$. The median length of hospital stay was 9 days (IQR 6 -14). Coagulopathic patients stayed in the hospital longer $\{12$ days (IQR 7 - 14) $\}$ than non-coagulopathic patients $\{8$ days (IQR $5-12)\}$. This showed statistical significance $(p=0.014)$. Eighteen patients $(12.9 \%)$ required ICU admission. Of these, 15 (83.3\%) had coagulopathy. The median duration of ICU stay was $2(<1-12)$ days. There was high probability of ICU admission in patients with coagulopathy $(\mathrm{OR}=5.5, \mathrm{p}=0.005)$ than in those with normal coagulation. Using Kaplan Meier curves and log rank test, we demonstrated significant difference in survival between patients with and those without coagulopathy $(\mathrm{p}=<0.001)$ (Figure 1)

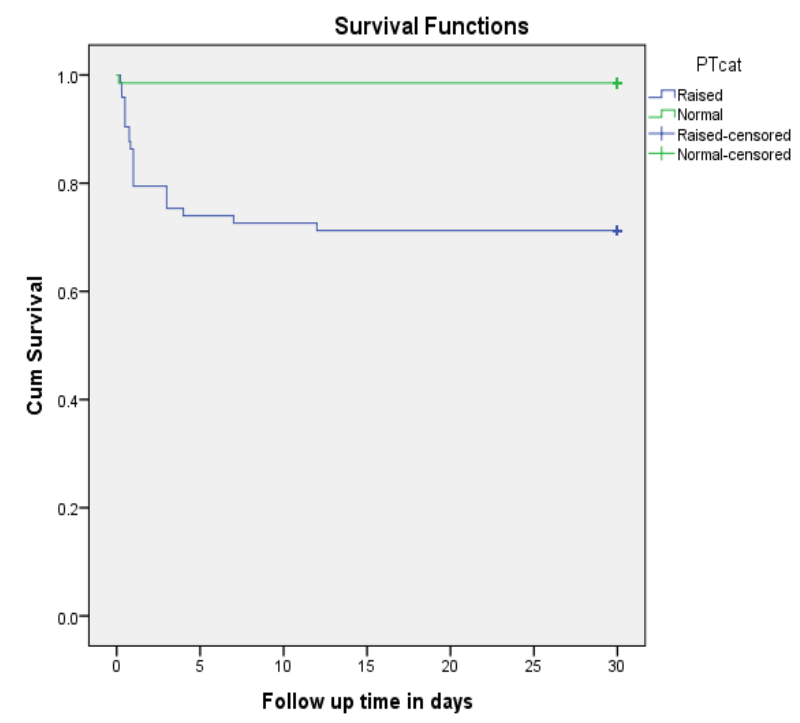

Figure 1: Comparative survival curves

\section{Discussion}

The incidence of coagulopathy of trauma was $52.1 \%$. This was high in comparison with other studies done in the West which range between 10 to $34 \%(2,3,5-7)$. In 
our study, we used abnormal PT as the marker of coagulation status. It has been shown that more patients have abnormal PT than PTT (5). Another explanation to the disparity could be due to the difference in the study design. Most studies have evaluated coagulopathy among all trauma patients including minor trauma while this study only looked at major trauma patients. Our study population had a majority of patients $(65 \%)$ with head and neck injuries. Some studies have documented a particularly higher incidence of coagulopathy of trauma (10). The median pre-hospital time was 4 hours (IQR 3 - 8.3). This is contrary to other countries with well organized pre-hospital care systems where the average time from injury to hospital admission is less than 70 minutes $(3,5,11)$. Low and Middle-income countries (LMICs) have been shown to bear a higher burden of trauma with $90 \%$ of all trauma deaths occurring in these countries. In addition, individuals who sustain injuries in LMICs are up to 6 times more likely to die than those in (High Income Countries) HICs (12). We speculate that there may be a higher incidence of coagulopathy of trauma contributing to the higher observed mortality in LMICs. An overwhelming majority of our patients were male (85.7\%) implying that males are the more often involved in trauma either because they the ones who are more likely to be involved in activities of fending for their families or because they are more aggressive in nature. The mean age of 29.4 years, with a majority $(40.7 \%)$ of the patients aged 20 to 29 years is comparable to local and international studies. The huge proportion $(77.8 \%)$ of patients in the working age group (19 - 49 years) shows the potential economic consequences of trauma. The pre-hospital delay signifies a loss in the therapeutic window, a vital time which could be utilized with possible reduction in morbidity and mortality. Although patients with longer pre-hospital delay had a higher incidence of coagulopathy, this did not reach statistical significance $(\mathrm{p}=0.573)$. Other authors have documented a clear relationship between hospital delay and the risk of coagulopathy of trauma $(1,3,5,6)$. The reason could because of the low numbers in our study. Coagulopathic patients had a significantly longer hospital stay $(\mathrm{p}=0.014)$. Several studies have made a similar observation $(3,4,6)$. We analyzed the length of hospital stay for the $118(84.2 \%)$ patients who did not die. Most of the mortalities in our study were early and involved the patients with coagulopathy. Coagulopathic patients who survived had a longer hospital stay since they required more care.

Most of the injuries were due to RTIs (55\%). Previous authors $(13,14)$ have documented RTIs as being responsible for over 40 percent of trauma mortalities. A majority of the patients recruited in the study had polytrauma since the eligibility criteria was an ISS above 15 . As observed by other authors $(5,15)$, patients with coagulopathy of trauma had a higher ISS $(p=0.018)$. Patients with ISS of 25 and above who are hypothermic and acidotic have been said to be universally coagulopathic. We did not do blood gas analysis in our study. Thirteen patients ( 8 of them with normal coagulation) developed AKI. The risk of developing AKI was lower among coagulopathic patients with $\mathrm{OR}=0.5(0.2-1.7)$. This was not statistically significant $(\mathrm{p}=0.286)$. Previous research has demonstrated an increased risk of developing AKI among coagulopathic patients which is in contrast to our findings $(4,6)$. More studies need to be done to evaluate and explain this finding.

A total of $32(22.9 \%)$ patients were transfused, two thirds $(68.8 \%)$ of whom had coagulopathy. There was a strong correlation between coagulopathy and need for blood transfusion with (OR 2.5 (1.1 - 5.7), $\mathrm{p}=0.032)$. Our study had similar findings to prior data (5).

The overall mortality was $22(15.7 \%)$. This is comparable to other studies in high volume dedicated trauma centers the West which have reported mortality rates of between 15 and $20 \%(2,3,5,6)$. This is a good statistic bearing in mind that we looked at major trauma patients only in a facility that is not a dedicated trauma centre. With shorter pre hospital delay, the mortality may have been lower. Nearly all the patients (95.5\%) who died had coagulopathy. There was a very strong correlation between coagulopathy and mortality (OR 26.7 (3.5 - 204.7), $\mathrm{p}<0.001)$. Multiple regressions showed that coagulation status, blood pressure and GCS were the variable that influenced outcome the most. Kaplan Meier survival curves demonstrated a significant difference in survival between the patients with and those without coagulopathy $(p<0.001)$. 
Majority of the deaths in patients with coagulopathy of trauma occur following hospital admission.

The limitations of this study were the delays in surgical treatment or undiagnosed intercurrent illnesses that may have affected the outcome.

In conclusion, the incidence of coagulopathy of trauma is high in major trauma patients and possibly higher in our set up. Determination of coagulation status in trauma patients on admission is a very important predictor of outcome. We suggest coagulation assay to be done in trauma patients on admission with an aim of improving their outcome.

\section{References}

1. Kapsch DN, Metzler M, Harrington M, et al. Fibrinolytic Response to Trauma. Surgery. 1984; 95:473-8.

2. Brohi K, Singh J, Heron M, et al. Acute Traumatic Coagulopathy. J Trauma .2003; 54:1127-30.

3. Brohi K, Cohen MJ, Ganter MT, et al. Acute Traumatic Coagulopathy: Initiated by Hypoperfusion: Modulated through the Protein C Pathway? Ann Surg 2007; 245:812-8.

4. Brohi K, Mitchell JC, Ross A D. Acute Coagulopathy of Trauma: Mechanism, Identification and Effect. Curr Opin Crit Care. 2007; 13:680-5

5. Macleod JBA, Lynn M, McKenney MG, et al. Early Coagulopathy Predicts Mortality in Trauma. J Trauma. 2003; 55:39-44.

6. Maegele M, Lefering R, Yucel N, et al. Early Coagulopathy in Multiple Injury: An Analysis from the German Trauma Registry on 8724 Patients. Injury. 2007; 38:298-304.

7. Rugeri L, Levrat A, David JS, et al. Diagnosis of Early Coagulation Abnormalities in Trauma Patients by Rotation Thrombelastography. J Thromb Haemost 2007; 5:289-95.

8. Schreiber MA. Damage Control Surgery. Crit Care Clin 2004; 20: 101-18.

9. Maegele M. Acute Traumatic Coagulopathy: Incidence, Risk Stratification and Therapeutic Options. World J Emerg Med. 2010; 1(1):12.

10. Gando S: Disseminated Intravascular Coagulation in Trauma Patients. Semin Thromb Hemost .2001, 27:585-92.
11. Stevens KA, Paruk F, Bachani AM, et al. Establishing Hospital-based Trauma Registry Systems: Lessons from Kenya. Injury. 2013; 44:S70-S74.

12. Organization WH, others. Injuries and Violence: the Facts. Inj Violence Facts [Internet]. 2010 [cited 2017 Mar 1]; Available from: https://www.cabdirect.org/cabdirect/abstract/201331 59250

13. Cothren CC, Biffl WL, Moore EE. Chapter 7.Trauma. In: Brunicardi FC, Andersen DK, Billiar TR, et al., editors. Schwartz's Principles of Surgery. 9th Ed. New York, NY: The McGraw-Hill Companies; 2010

14. Odero W, Khayesi M, Heda PM. Road Traffic Injuries in Kenya: Magnitude, Causes and Status of Intervention. Inj Control Saf Pomot. 2003;10:53

15. Keimowitz RM, Annis BL. Disseminated Intravascular Coagulation Associated with Massive Brain Injury. J Neurosurg. 1973;39:178-80 